\title{
The Conservation of Allostery in C. Elegans UDP-Glucose Dehydrogenase
}

Weston McDonald, Nicholas Keul, Zachary Wood, University of Georgia

The human enzyme UDP-Glucose Dehydrogenase (hUGDH) catalyzes the $\mathrm{NAD}^{+}$-dependent reaction of UDP-Glucose to UDP-Glucuronic acid. The product is essential to glucuronidation, a detoxification pathway of drugs in the human body. This process enhances the rate at which drugs are excreted, and leads to many drugs failing clinical trials. hUGDH catalysis is dependent on its hexameric structure, which is allosterically regulated by a downstream feedback inhibitor, UDP-Xylose. The binding of UDP-Xylose in the active site induces the formation of a distinct inactive conformation of the enzyme through the translation of a conserved allosteric switch. Identifying ways to inhibit UGDH by studying the evolution of this switch, and the cavities it occupies, could provide ways to influence glucuronidation. Bioinformatics studies identified $C$. elegans UGDH (cUGDH) to be one of the more divergent versions of UGDH that still conserve the allosteric switch. Structural studies with cUGDH show that UDP-Xylose induces the same conformational change in the enzyme. Sedimentation velocity studies show that UDP-Xylose stabilizes the cUGDH hexamer in solution. These results suggest that the allosteric mechanism is preserved between the divergent enzymes. However, steady-state kinetic studies show that the affinity for UDP-Xylose in cUGDH is reduced by more than an order of magnitude. Future studies will aim at solving which structural changes in the functional allosteric switch causes a lesser affinity in cUGDH. I am currently refining the X-Ray crystallographic data of UDP-Xylose-bound cUGDH, and plan to overlay the refined structure to discover any observable differences. 